[Article]

\title{
层间阴离子对四元水滑石超分子作用力的影响
}

\author{
胡军施 炜倪哲明* 刘 娇 薛继龙
}

(浙江工业大学化学工程与材料学院, 先进催化材料实验室, 杭州 310032)

\begin{abstract}
摘要: 构建一价阴离子 $\left(\mathrm{X}=\mathrm{F}^{-}, \mathrm{Cl}^{-}, \mathrm{Br}^{-}, \mathrm{I}^{-}, \mathrm{NO}_{3}^{-}, \mathrm{OH}^{-}\right)$插层铜锌镁铝四元水滑石(CuZnMgAl-X)周期性计算模 型, 采用密度泛函理论(DFT), 选取 CASTEP程序模块, 对体系进行几何全优化, 从结合能、结构参数、Mulliken 布居、氢键布居、态密度等角度研究了不同层间阴离子的分布形态以及其对主客体间超分子作用的影响. 结果 表明, 随着CuZnMgAl-X体系层间阴离子电负性的减弱, 电子逐渐从层间阴离子向层板发生转移, 主客体间静 电作用力逐渐减小, 氢键强度逐渐降低, 禁带宽度逐渐变窄, 体系电子更易向高能级发生跃迁, 稳定性逐渐下 降. 此外, Cu 的掺杂使得 CuZnMgAl-X 体系的价带顶向高能量处发生偏移, 禁带宽度较传统水滑石体系更窄, 稳定性更低, 进一步解释了含铜水滑石较难合成的原因.
\end{abstract}

关键词：密度泛函理论；铜锌镁铝四元水滑石；不同层间阴离子； 超分子作用力 中图分类号: 0641

\section{Influence of Interlayer Anion on Supermolecular Interaction in Quaternary Hydrotalcites}

\author{
HU Jun SHI Wei NI Zhe-Ming* LIU Jiao XUE Ji-Long \\ (Laboratory of Advanced Catalytic Materials, College of Chemical Engineering and Materials Science, \\ Zhejiang University of Technology, Hangzhou 310032, P. R. China)
}

\begin{abstract}
A periodic interaction model with different intercalated anions $\left(\mathrm{X}=\mathrm{F}^{-}, \mathrm{Cl}^{-}, \mathrm{Br}^{-}, \mathrm{I}^{-}, \mathrm{NO}_{3}^{-}, \mathrm{OH}^{-}\right)$is proposed for the CuZnMgAl quaternary hydrotalcites (CuZnMgAl-X). Based on density functional theory, the CuZnMgAl-X geometry was optimized using the CASTEP program. The distribution of anions in the interlayer, and the supra-molecular interaction between host layer and guest anions were investigated by analyzing binding energies, geometric parameters, Mulliken populations, hydrogen-bonding and densities of states. A decreased electronegativity of interlayer anion caused a transfer of charge from guest anions to host layer and a gradual decrease in the strength of electrostatic interaction and hydrogen bonding. The system band gap narrowed, electrons transferred to higher energy levels more easily, and the overall stability of the system decreased. The Cu dopant caused a deviation in CuZnMgAl-X valence band to high energies. Compared with traditional layered double hydroxides, the band gap narrowed and stability decreased, accounting for the difficulty in preparing copper-containing hydrotalcites.
\end{abstract}

Key Words: Density functional theory; Cu-Zn-Mg-Al quaternary hydrotalcite; Different intercalated anions; Supra-molecular interaction

1 引 言

层状双金属氢氧化物(LDHs) 又称水滑石类化 合物或阴离子粘土, 1 , 它是一类由带正电的金属氢
氧化物为主体层板, 层间具有可交换阴离子的层柱 状化合物, 由于该类化合物主体层板金属的可调控 性以及层间阴离子的可交换性, 使其在催化、吸附、

Received: November 9, 2012; Revised: January 7, 2013; Published on Web: January 7, 2013.

"Corresponding author. Email: jchx@zjut.edu.cn; Tel: +86-13858123256.

The project was supported by the National Natural Science Foundation of China (51002137).

国家自然科学基金(51002137)资助项目

(C) Editorial office of Acta Physico-Chimica Sinica 
阻然、离子交换等领域具有广泛的应用前景. ${ }^{1-5}$

随着水滑石制备技术的进步及其应用领域的 拓宽, 层间阴离子调变以及多元水滑石的合成越来 越受到人们的关注. ${ }^{6-9}$ Parida 和 Mohapatra $^{6}$ 曾合成了 一系列不同阴离子插层的 ZnFe-LDHs, 比较了它们 对于含氮染料的光降解活性, 发现不同阴离子插层 的 ZnFe-LDHs 之间显示出了不同的催化性能; 王松 林等 ${ }^{7}$ 研究了不同阴离子插层的 MgAl-LDHs 阻然 剂, 结果表明层间阴离子的不同会影响 LDHs 的阻 燃效果; 吴健松等 ${ }^{8}$ 采用水热法合成了排列规整、粒 度均匀的 $\mathrm{CuZnMgAl}$ 四元水滑石, 探讨了适宜的合

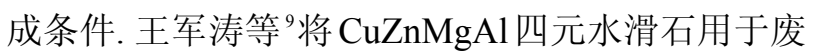
水中 $\mathrm{Pb}^{2+}$ 的吸附, 性能优越. 但是, 由于 LDHs 层间阴 离子排布的无序性和多元水滑石层板构成的复杂 性, 目前要从实验角度精确探求阴离子在多元 LDHs 层间的排布情况、电子性质以及主客体超分 子作用还存在相当大的困难, 有必要引入计算机模 拟技术.

密度泛函理论 $(\mathrm{DFT})^{10,11}$ 是一种采用电子密度分 布函数来研究多电子体系电子结构的量子力学方 法, 目前已被广泛应用于研究水滑石体系的结构参 数、成键状况、作用能、电子密度等性质. Fraccarollo 等 ${ }^{12}$ 曾以 MgAl-LDHs 为主体, 采用混合密度泛函 B3LYP 方法讨论了 $\mathrm{CO}_{3}^{2-} 、 \mathrm{NO}_{3}^{-} 、 \mathrm{Cl}^{-} 、 \mathrm{I}^{-} 、 \mathrm{I}_{3}^{-}$插层 LDHs 的层间排布、结构参数, 并在此基础上与实验值进 行比较, 吻合性较好. 本课题组曾利用 DFT 总结了 一价阴离子插层 MgAl-LDHs 的相互作用规律及微 观结构性质; ${ }^{13}$ 此外, 还曾探讨了 CuZnMgAl-LDHs 的层板排布方式和结构稳定性. ${ }^{14}$ 基于以上原因, 本 文采用密度泛函理论, 构建了一系列一价阴离子 $\left(\mathrm{F}^{-}, \mathrm{Cl}^{-}, \mathrm{Br}^{-}, \mathrm{I}^{-}, \mathrm{OH}^{-}, \mathrm{NO}_{3}^{-}\right)$插层的铜锌镁铝四元水滑 石模型, 从结合能、结构参数、Mulliken 布居、氢键布 居、态密度等角度研究了层间阴离子的分布形态以 及其对主客体间超分子作用的影响, 为多元水滑石 的阴离子插层改性提供理论参考.

\section{2 计算模型与方法}

以 $2 \mathrm{H}$ 堆积模式 ${ }^{1,2}$ 构建了铜锌镁铝四元水滑石 主体层板 $\left[\mathrm{Cu}_{2} \mathrm{Zn}_{2} \mathrm{Mg}_{2} \mathrm{Al}_{2}(\mathrm{OH})_{16}\right] \mathrm{X}_{2}$, 记作 $\mathrm{CuZnMgAl}-$ $\mathrm{X}$, 其中 $\mathrm{X}$ 为以下几种一价阴离子: $\mathrm{F}^{-} 、 \mathrm{Cl}^{-} 、 \mathrm{Br}^{-} 、 \mathrm{I}^{-}$、 $\mathrm{NO}_{3}^{-} 、 \mathrm{OH}^{-}$, 将层间阴离子分为两类: $\mathrm{A}$ 类为单原子层 间阴离子, $\mathrm{B}$ 类为多原子层间阴离子. 采用先前工作 中 ${ }^{13,14}$ 证实的 $\mathrm{Cu}$ 在晶胞顶点, $\mathrm{Al}$ 在晶胞中心的层板
排布方式, 选取层间阴离子处于较稳定的 Hcp-Al 位 且位于层板间中心位置的构型为初始模型, 如图 1 所示.

通过 Material Studio 5.5 软件中 CASTEP 模块, ${ }^{15}$ 利用局域密度近似(LDA)泛函下的 CA-PZ 基组 ${ }^{16}$ 对 模型进行几何全优化. 其中, 原子电子采用超软赝 势, ${ }^{17}$ 截止能量为 $330.0 \mathrm{eV}$, 自洽场计算的误差为 $2 \times$ $10^{-6} \mathrm{eV} \cdot \mathrm{atom}^{-1}$, 能带结构在布里渊区 $k$ 矢量的选取 为 $4 \times 4 \times 1$, 基态能量选用 Pulay 密度混合算法, ${ }^{18}$ 整体 电荷数为 0 , 电子的计算采用自旋极化方法, 同时优 化晶胞, 其它参数设置为程序的默认值. 图 2 列出了 所有体系最终优化后的结构和氢键分布图.

\section{3 结果与讨论}

\section{1 结合能}

为估计结构的稳定性, 现定义 $\mathrm{CuZnMgAl}-\mathrm{X}$ 体 系的结合能 $\Delta E_{\mathrm{CuZnMgAl}-\mathrm{X}}$ 为:

$$
\begin{aligned}
\Delta E_{\mathrm{CuZnMgAl}-\mathrm{X}}= & E_{\mathrm{CuZnMgAl}-\mathrm{X}}-\left(16 E_{\mathrm{H}}+16 E_{\mathrm{O}}+2 E_{\mathrm{X}}+2 E_{\mathrm{Cu}}+\right. \\
& \left.2 E_{\mathrm{Zn}}+2 E_{\mathrm{Mg}}+2 E_{\mathrm{Al}}\right)
\end{aligned}
$$

其中 $E_{\mathrm{CuZnMgAl}-\mathrm{X}}$ 为优化后 $\mathrm{CuZnMgAl}-\mathrm{X}$ 体系的能 量, $E_{\mathrm{N}}=-25295.7 \mathrm{~kJ} \cdot \mathrm{mol}^{-1} 、 E_{\mathrm{H}}=-1169.2 \mathrm{~kJ} \cdot \mathrm{mol}^{-1} 、 E_{\mathrm{O}}=$ $-41364.3 \mathrm{~kJ} \cdot \mathrm{mol}^{-1} 、 E_{\mathrm{F}}=-63301.9 \mathrm{~kJ} \cdot \mathrm{mol}^{-1} 、 E_{\mathrm{Cl}}=-39180.6$ $\mathrm{kJ} \cdot \mathrm{mol}^{-1} 、 E_{\mathrm{Br}}=-35232.9 \mathrm{~kJ} \cdot \mathrm{mol}^{-1} 、 E_{\mathrm{l}}=-30233.4 \mathrm{~kJ} \cdot$ $\mathrm{mol}^{-1} 、 E_{\mathrm{Cu}}=-129759.8 \mathrm{~kJ} \cdot \mathrm{mol}^{-1} 、 E_{\mathrm{Zn}}=-165066.0 \mathrm{~kJ} \cdot$ $\mathrm{mol}^{-1} 、 E_{\mathrm{Mg}}=-93770.7 \mathrm{~kJ} \cdot \mathrm{mol}^{-1} 、 E_{\mathrm{Al}}=-5114.7 \mathrm{~kJ} \cdot$ $\mathrm{mol}^{-1}$, 分别为各原子的能量, 体系的结合能结果列 于表 1 中.

从表 1 可以看出, CuZnMgAl-X 体系的总势 能绝对值顺序为: $\mathrm{CuZnMgAl}-\mathrm{NO}_{3}>\mathrm{CuZnMgAl}-\mathrm{F}>$ $\mathrm{CuZnMgA1-OH}>\mathrm{CuZnMgA1-Cl}>\mathrm{CuZnMgAl}-\mathrm{Br}>$ $\mathrm{CuZnMgAl}-\mathrm{I}$, 与层间阴离子电负性大小相一致. 结合 能绝对值顺序为: $\mathrm{CuZnMgAl}-\mathrm{NO}_{3}>\mathrm{CuZnMgAl}-\mathrm{OH}>$ $\mathrm{CuZnMgAl-F}>\mathrm{CuZnMgA1-Cl}>\mathrm{CuZnMgA1-Br}>$ $\mathrm{CuZnMgAl}-\mathrm{I}, \mathrm{B}$ 类水滑石的结合能均大于 A 类水滑 石, 且 $\mathrm{A}$ 类和 $\mathrm{B}$ 类水滑石的结合能均随着层间阴离 子电负性的减弱而降低, 体系稳定性逐渐下降.

\section{2 结构参数}

表 2 列出了优化后 $\mathrm{CuZnMgAl-X}$ 体系的结构参 数. 晶胞参数 $a$ 值和 $b$ 值的大小主要归因于层板金 属离子半径的影响, 即在 $\mathrm{CuZnMgAl}-\mathrm{X}$ 体系中, $a 、 b$ 主要与晶胞棱上的 $\mathrm{Cu}-\mathrm{O} 、 \mathrm{Zn}-\mathrm{O} 、 \mathrm{Mg}-\mathrm{O} 、 \mathrm{Al}-\mathrm{O}$ 键长大小相关. ${ }^{2}$ 从表 2 可以看出, 对于 $\mathrm{A}$ 类水滑石, $a 、 b$ 值随着层间阴离子电负性的减弱而增大, 键长 


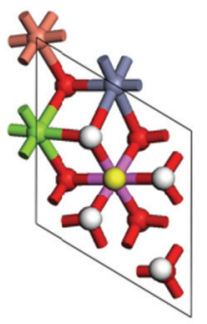

(a) CuZnMgAl-F, Cl, Br, I

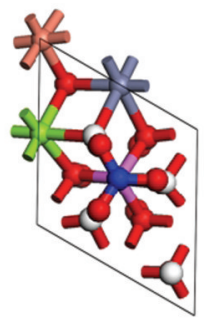

(b) CuZnMgAl- $\mathrm{NO}_{3}$
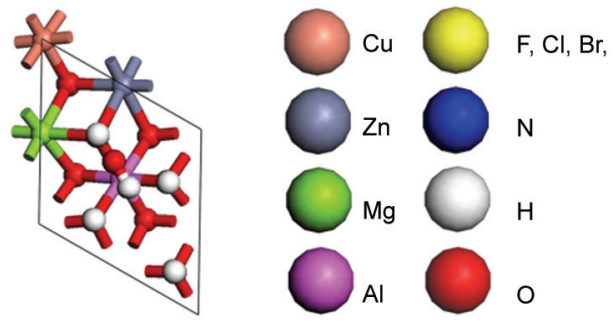

(c) $\mathrm{CuZnMgAl}-\mathrm{OH}$

图 $1 \mathrm{CuZnMgAl-X}$ 主客体计算模型

Fig.1 Host-guest calculation models of CuZnMgAl-X

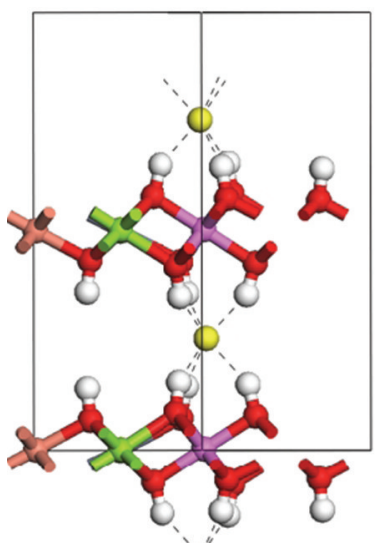

(a) CuZnMgAl-F, Cl, Br, I

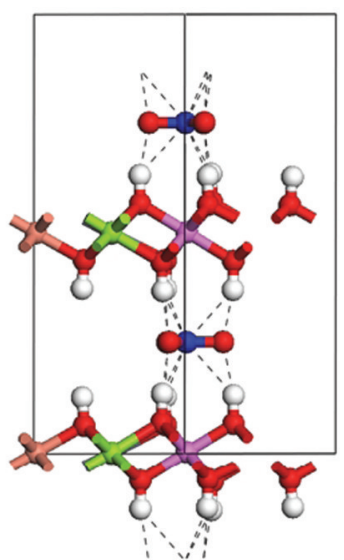

(b) $\mathrm{CuZnMgAl}-\mathrm{NO}_{3}$

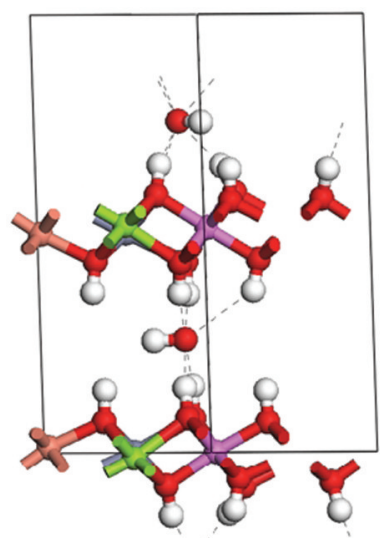

(c) CuZnMgAl-OH

图 2 CuZnMgAI-X 优化构型和层间氢键分布情况

Fig.2 Geometry structures and distribution of hydrogen-bonding between layers of CuZnMgAl-X

$l_{\mathrm{Cu}-0} 、 l_{\mathrm{Mg}-\mathrm{O}} 、 l_{\mathrm{Zn}-\mathrm{o}}$ 随着层间阴离子电负性的减弱而呈 减小趋势, $l_{\mathrm{Al}-\mathrm{O}}$ 随着层间阴离子电负性的减弱而增 大; 对于 $\mathrm{B}$ 类水滑石, $a 、 b$ 值随着层间阴离子电负性 的减弱而减小, $l_{\mathrm{Cu}-0} 、 l_{\mathrm{Mg}-0} 、 l_{\mathrm{Zn}-\mathrm{O}}$ 随着层间阴离子电负 性的减弱而增大, $l_{\mathrm{Al}-\mathrm{o}}$ 随着层间阴离子电负性的减 弱而减小, 故可认为 $l_{\mathrm{Al}-\mathrm{o}}$ 在决定层板 $a 、 b$ 大小中起 决定性作用.

晶胞参数 $c$ 值(层间距 $d_{c}=0.5 c$ ) 为层板厚度和层 间通道的加和, 其不仅受到层板金属离子半径的影 响, 还受到主客体间作用力大小的影响. ${ }^{2}$ 由表 2 可 知, 层间距会随着层间阴离子的不同而改变. 对于 $\mathrm{A}$ 类水滑石, 层间距随着层间阴离子电负性的减弱而 增大, 这主要是由于随着电负性的减弱, 体系结合
能逐渐降低(数据见表 1), 主客体作用力逐渐减小, 从而使得 $c$ 值增大; 对于 $\mathrm{B}$ 类水滑石, 层间距反而随 着层间阴离子电负性的减弱而减小, 这可能是由于 $\mathrm{NO}_{3}^{-}$基团较大, 所产生的空间效应所造成的.

\subsection{Mulliken 布居分析}

Mulliken 布居是 Mulliken ${ }^{20}$ 提出的一种基于电 子轨道来定量描述体系电荷分布情况的方法, 它常 用于分析相同基组下体系的电荷变化趋势, 间接地 讨论分子内相互作用力的强弱. 因此, 为了进一步 研究 CuZnMgAl-X 体系中主体层板与客体阴离子 间的作用力变化, 表 3、表 4、表 5 分别列出了各体系 的键布居、原子电荷布居和原子轨道布居(对上下层 板、层间阴离子、各原子的轨道布居作了平均处理).

表 $1 \mathrm{CuZnMgAl-X}$ 的结合能 $(\Delta E)$

Table 1 Binding energy $(\Delta E)$ of $\mathrm{CuZnMgAl}-\mathrm{X}$

\begin{tabular}{clccc}
\hline Type & \multicolumn{1}{c}{ System } & $E_{\text {CuznMgA-X-LDH }}\left({\left.\mathrm{kJ} \cdot \mathrm{mol}^{-1}\right)}\right)$ & $\Delta E_{\text {CunngAl-X-LDHs }} /\left(\mathrm{kJ} \cdot \mathrm{mol}^{-1}\right)$ & Electronegativity of anion $^{19}$ \\
\hline $\mathrm{A}$ & CuZnMgAl-F & -1618392.7050 & -23830.5050 & 3.98 \\
& CuZnMgAl-Cl & -1569795.3548 & -23475.7548 & 3.16 \\
& CuZnMgAl-Br & -1561805.6041 & -23381.4354 & 2.96 \\
& CuZnMgAl-I & -1551675.2222 & -23250.0222 & 2.66 \\
B & CuZnMgAl-NO & -1766735.6609 & -28336.8190 & 4.58 \\
& CuZnMgAl-OH & -1577915.8400 & -24890.4400 & 3.51 \\
\hline
\end{tabular}


表 $2 \mathrm{CuZnMgAl}-\mathrm{X}$ 的晶胞参数和键长数据

Table 2 Lattice parameters and bond length data of CuZnMgAl-X

\begin{tabular}{|c|c|c|c|c|c|c|}
\hline \multirow{2}{*}{ System } & \multicolumn{2}{|c|}{ Lattice length/nm } & \multicolumn{4}{|c|}{ Bond length/nm } \\
\hline & $a, b$ & $c$ & $l_{\mathrm{Al}-\mathrm{O}}$ & $l_{\mathrm{Cu}-\mathrm{O}}$ & $l_{\mathrm{Mg}-\mathrm{O}}$ & $\overline{l_{\mathrm{zn}-\mathrm{O}}}$ \\
\hline $\mathrm{CuZnMgAl-F}$ & 1.2094 & 1.3502 & 0.1882 & 0.2081 & 0.2067 & 0.2090 \\
\hline $\mathrm{CuZnMgAl-Cl}$ & 1.2120 & 1.5383 & 0.1884 & 0.2074 & 0.2067 & 0.2086 \\
\hline $\mathrm{CuZnMgAl}-\mathrm{Br}$ & 1.2122 & 1.6201 & 0.1884 & 0.2075 & 0.2065 & 0.2086 \\
\hline CuZnMgAl-I & 1.2126 & 1.6879 & 0.1885 & 0.2071 & 0.2065 & 0.2083 \\
\hline $\mathrm{CuZnMgAl}-\mathrm{NO}_{3}$ & 1.2176 & 1.5277 & 0.1893 & 0.2072 & 0.2067 & 0.2084 \\
\hline $\mathrm{CuZnMgAl-OH}$ & 1.2107 & 1.3687 & 0.1887 & 0.2084 & 0.2078 & 0.2085 \\
\hline
\end{tabular}

$a, b:$ the sum of lattice parameters $a$ and $b$

从图 2 和表 3 数据可以看出, 层板上的金属离 子和氧原子之间不仅存在着离子键, 同时还具有共 价键. 层间阴离子与层板金属相距较远, 它们之间 主要是较弱的静电作用力, 总体表现为静电吸引. 从 表 4 数据可以看出, 当阴离子插层后, 其电荷布居由 $-1.000 e$ 分别降低为 $-0.6800 e 、-0.6500 e 、-0.6300 e$ 、 $-0.6300 e 、-0.7500 e 、-0.5800 e$, 层板布居由 $1.000 e$ 分别降低为 $0.6906 e 、 0.6552 e 、 0.6296 e 、 0.6268 e$ 、 $0.7698 e 、 0.5700 e$. 由此可见, 部分电子由层间客体阴 离子向主体层板发生了转移. 对于 $\mathrm{A}$ 类和 $\mathrm{B}$ 类水滑 石, 层板及层间阴离子电荷均随着层间阴离子电负
性的减弱而减小, 说明其静电作用力逐渐减弱.

表 5 中列出了 $\mathrm{CuZnMgAl}-\mathrm{X}$ 体系的原子轨道布 居, 定量地分析了不同原子轨道对化学键成键的贡 献. 各原子轨道的初始电子分布为: $\mathrm{H}$ 为 $1 s^{1}, \mathrm{O}$ 为 $2 s^{2} 2 p^{4}, \mathrm{Al}$ 为 $3 s^{2} 3 p^{1}, \mathrm{Mg}$ 为 $2 p^{6} 3 s^{2}, \mathrm{Cu}$ 为 $4 s^{1} 3 d^{10}, \mathrm{Zn}$ 为 $4 s^{2} 3 d^{10}$, 优化后层板中 $\mathrm{Cu} 、 \mathrm{Zn} 、 \mathrm{Mg} 、 \mathrm{Al}$ 的 $s$ 轨道上的 电子发生了大幅降低, 说明 $s$ 轨道上的电子发生了 一定程度的离域. 同时, $\mathrm{Mg} 、 \mathrm{Al}$ 的 $p$ 轨道上的电子被 氧原子定域, 这说明了 $\mathrm{CuZnMgAl}-\mathrm{X}$ 层板中的金属 离子和羟基间同时存在着离子键和共价键, 与表 3 Mulliken键布居的分析结果相一致.

表 $3 \mathrm{CuZnMgAl}-\mathrm{X}$ 的 Mulliken 成键布居(e)

Table 3 Mulliken bond population (e) of CuZnMgAl-X

\begin{tabular}{lccccc}
\hline \multicolumn{1}{c}{ System } & $Q(\mathrm{Cu}-\mathrm{O})$ & $Q(\mathrm{Al}-\mathrm{O})$ & $Q(\mathrm{Mg}-\mathrm{O})$ & $Q(\mathrm{Zn}-\mathrm{O})$ & $Q(\mathrm{H}-\mathrm{O})$ \\
\hline CuZnMgAl-F & 0.2425 & 0.3633 & -0.9525 & 0.2075 & 0.5856 \\
CuZnMgAl-Cl & 0.2317 & 0.3667 & -0.9592 & 0.2333 & 0.5900 \\
CuZnMgAl-Br & 0.2392 & 0.3683 & -0.9633 & 0.2208 & 0.5975 \\
CuZnMgAl-I & 0.2392 & 0.3683 & -0.9650 & 0.2192 & 0.5969 \\
CuZnMgAl-NO $_{3}$ & 0.2317 & 0.3700 & -0.9675 & 0.2267 & 0.6038 \\
CuZnMgAl-OH & 0.2367 & 0.3700 & -0.9033 & 0.2150 & 0.5763 \\
\hline
\end{tabular}

表 $4 \mathrm{CuZnMgAl-X}$ 的 Mulliken 原子电荷布居 $(e)$

Table 4 Mulliken atomic population (e) of CuZnMgAl-X

\begin{tabular}{lcccccccc}
\hline \multicolumn{1}{c}{ System } & $Q(\mathrm{H})$ & $Q(\mathrm{O})$ & $Q(\mathrm{Mg})$ & $Q(\mathrm{Al})$ & $Q(\mathrm{Zn})$ & $Q(\mathrm{Cu})$ & $Q($ layer $)$ & $Q(\mathrm{X})$ \\
\hline CuZnMgAl-F & 0.3988 & -0.9581 & 1.9550 & 1.5500 & 1.0000 & 0.6600 & 0.6906 & -0.6800 \\
CuZnMgAl-Cl & 0.3925 & -0.9556 & 1.9700 & 1.5600 & 0.9500 & 0.6800 & 0.6552 & -0.6500 \\
CuZnMgAl-Br & 0.3850 & -0.9513 & 1.9700 & 1.5600 & 0.9700 & 0.6600 & 0.6296 & -0.6300 \\
CuZnMgAl-I $_{\text {CuZnMgAl-NO }}$ & 0.3834 & -0.9488 & 1.9700 & 1.5600 & 0.9600 & 0.6600 & 0.6268 & -0.6300 \\
CuZnMgAl-OH & 0.3994 & -0.9563 & 1.9800 & 1.5400 & 1.0150 & 0.6900 & 0.7698 & -0.7500 \\
\hline
\end{tabular}

表 $5 \mathrm{CuZnMgAl-X}$ 的原子轨道布居

Table 5 Atomic orbital populations of CuZnMgAl-X

\begin{tabular}{|c|c|c|c|c|c|c|c|}
\hline System & $\mathrm{H}$ & $\mathrm{O}$ & $\mathrm{Cu}$ & $\mathrm{Zn}$ & $\mathrm{Mg}$ & $\mathrm{Al}$ & $X$ \\
\hline $\mathrm{CuZnMgAl-F}$ & $1 s^{0.62}$ & $2 s^{1.86} 2 p^{5.10}$ & $4 s^{0.52} 3 d^{9.37}$ & $4 s^{0.34} 3 d^{9.99}$ & $3 s^{0.33} 2 p^{5.72}$ & $3 s^{0.56} 3 p^{0.89}$ & $2 s^{1.98} 2 p^{5.70}$ \\
\hline $\mathrm{CuZnMgAl}-\mathrm{Cl}$ & $1 s^{0.61}$ & $2 s^{1.86} 2 p^{5.10}$ & $4 s^{0.51} 3 d^{9.37}$ & $4 s^{0.40} 3 d^{9.99}$ & $3 s^{0.32} 2 p^{5.71}$ & $3 s^{0.55} 3 p^{0.89}$ & $3 s^{1.97} 3 p^{5.68}$ \\
\hline $\mathrm{CuZnMgAl}-\mathrm{Br}$ & $1 s^{0.62}$ & $2 s^{1.86} 2 p^{5.10}$ & $4 s^{0.51} 3 d^{9.37}$ & $4 s^{0.38} 3 d^{9.99}$ & $3 s^{0.32} 2 p^{5.71}$ & $3 s^{0.55} 3 p^{0.90}$ & $4 s^{1.97} 4 p^{5.66}$ \\
\hline CuZnMgAl-I & $1 s^{0.62}$ & $2 s^{1.86} 2 p^{5.10}$ & $4 s^{0.51} 3 d^{9.37}$ & $4 s^{0.37} 3 d^{9.99}$ & $3 s^{0.32} 2 p^{5.71}$ & $3 s^{0.55} 3 p^{0.90}$ & $5 s^{2.00} 5 p^{5.63}$ \\
\hline $\mathrm{CuZnMgAl}-\mathrm{NO}_{3}$ & $1 s^{0.62}$ & $2 s^{1.85} 2 p^{5.10}$ & $4 s^{0.53} 3 d^{9.38}$ & $4 s^{0.34} 3 d^{9.99}$ & $3 s^{0.33} 2 p^{5.77}$ & $3 s^{0.55} 3 p^{0.90}$ & $2 s^{1.22} 2 p^{3.24} / 2 s^{1.84} 2 p^{4.59}$ \\
\hline $\mathrm{CuZnMgAl-OH}$ & $1 s^{0.60}$ & $2 s^{1.85} 2 p^{5.10}$ & $4 s^{0.52} 3 d^{9.37}$ & $4 s^{0.35} 3 d^{9.99}$ & $3 s^{0.33} 2 p^{5.69}$ & $3 s^{0.55} 3 p^{0.91}$ & $2 s^{1.89} 2 p^{5.10} / 1 s^{0.60}$ \\
\hline
\end{tabular}


此外, 层间阴离子的初始原子轨道分别为: $\mathrm{F}$ 为 $2 s^{2} 2 p^{5}, \mathrm{Cl}$ 为 $3 s^{2} 3 p^{5}, \mathrm{Br}$ 为 $4 s^{2} 4 p^{5}$, I 为 $5 s^{2} 5 p^{5}, \mathrm{~N}$ 为 $2 s^{2} 2 p^{3}, \mathrm{H}$ 为 $1 s^{1}, \mathrm{O}$ 为 $2 s^{2} 2 p^{4}$. 在 $\mathrm{CuZnMgAl}-\mathrm{X}$ 体系 中, 优化后不同层间阴离子的原子轨道布居分别 为: $\mathrm{F}^{-}\left(2 s^{1.98} 2 p^{5.70}\right), \mathrm{Cl}^{-}\left(3 s^{1.97} 3 p^{5.68}\right), \mathrm{Br}^{-}\left(4 s^{1.97} 4 p^{5.66}\right), \mathrm{I}^{-}$ $\left(5 s^{2.00} 5 p^{5.63}\right), \mathrm{NO}_{3}^{-}\left(\mathrm{N} 2 s^{1.22} 2 p^{3.24} 、 \mathrm{O} 2 s^{1.84} 2 p^{4.59}\right), \mathrm{OH}^{-}(\mathrm{O}$ $\left.2 s^{1.89} 2 p^{5.10} 、 \mathrm{H} 1 s^{0.60}\right)$. 由此可见, 大部分层间阴离子 $s$ 轨 道上的电子被定域, 而层间阴离子 $p$ 轨道上的电子 则呈现出了轻度的离域现象. 层板金属 $\mathrm{Mg} 、 \mathrm{Al}$ 的 $p$ 轨道和层间阴离子的 $s$ 轨道对整个体系的离子键部 分贡献较大, 而层板金属阳离子的 $s$ 轨道和层间阴 离子的 $p$ 轨道对体系的共价键部分贡献较大.

\section{4 氢键布居分析}

氢键会对水滑石的微观结构及稳定性造成较 大影响, 它是一种广泛存在的分子间弱作用力, 它 是一种特殊的分子间或分子内作用, 其键长、键角 和方向性等各个方面都可以在相当大的范围内变 化, 具有一定的适应性和灵活性. ${ }^{21-23}$ 一般情况下, 氢 键的数目越多, 键长越短, 键角越接近 $180^{\circ}$, 氢键的 强度越强.

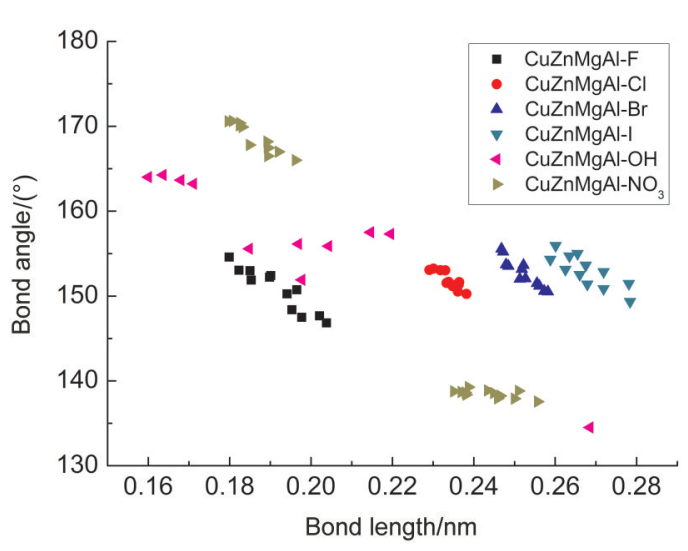

图 $3 \mathrm{CuZnMgAl}-\mathrm{X}$ 中氢键键角与键长的关系

Fig.3 Relationship between bond angle and bond length of hydrogen-bonding in CuZnMgAl-X

表 6 和图 3 分别列出了 $\mathrm{CuZnMgAl}-\mathrm{X}$ 体系的氢 键布居及分布图. 可以看出, CuZnMgAl-X体系中电 负性较强的层间阴离子与层板多个羟基上的氢原 子形成了多重氢键, 故该体系存在复杂的氢键网 络. 结合表 6 数据和图 3 可以看出以下几个特征: (1) 对于 $\mathrm{A}$ 类水滑石和 $\mathrm{B}$ 类水滑石, 氢键强度均随着层 间阴离子电负性的减弱而降低, 氢键强度顺序为:

表 $6 \mathrm{CuZnMgAI-X}$ 的氢键参数

Table 6 Hydrogen bond parameters of CuZnMgAl-X

\begin{tabular}{|c|c|c|c|c|c|c|c|c|c|c|c|}
\hline \multicolumn{2}{|c|}{$\mathrm{CuZnMgAl-F}$} & \multicolumn{2}{|c|}{$\mathrm{CuZnMgAl}-\mathrm{Cl}$} & \multicolumn{2}{|c|}{$\mathrm{CuZnMgAl}-\mathrm{Br}$} & \multicolumn{2}{|c|}{ CuZnMgAl-I } & \multicolumn{2}{|c|}{$\mathrm{CuZnMgAl}-\mathrm{OH}$} & \multicolumn{2}{|c|}{$\mathrm{CuZnMgAl}-\mathrm{NO}_{3}$} \\
\hline$l_{\mathrm{O}-\mathrm{H}} / \mathrm{nm}$ & $\theta_{\mathrm{H}-\mathrm{O}-\mathrm{H}} /\left(^{\circ}\right)$ & $l_{\mathrm{O}-\mathrm{H}} / \mathrm{nm}$ & $\theta_{\mathrm{H}-\mathrm{O}-\mathrm{H}} /\left(^{\circ}\right)$ & $l_{\mathrm{O}-\mathrm{H}} / \mathrm{nm}$ & $\theta_{\mathrm{H}-\mathrm{O}-\mathrm{H}} /\left({ }^{\circ}\right)$ & $l_{\mathrm{O}-\mathrm{H}} / \mathrm{nm}$ & $\theta_{\mathrm{H}-\mathrm{O}-\mathrm{H}} /\left(^{\circ}\right)$ & $l_{\mathrm{O}-\mathrm{H}} / \mathrm{nm}$ & $\theta_{\mathrm{H}-\mathrm{O}-\mathrm{H}} /\left({ }^{\circ}\right)$ & $l_{\mathrm{O}-\mathrm{H}} / \mathrm{nm}$ & $\theta_{\mathrm{H}-\mathrm{O}-\mathrm{H}} /\left(^{\circ}\right)$ \\
\hline 0.1823 & 153.05 & 0.2301 & 153.22 & 0.2485 & 153.53 & 0.2655 & 155.00 & 0.2146 & 157.50 & 0.1831 & 169.92 \\
\hline 0.1850 & 152.99 & 0.2350 & 151.27 & 0.2555 & 151.50 & 0.2601 & 155.94 & 0.1680 & 163.66 & 0.2387 & 139.27 \\
\hline 0.1953 & 148.38 & 0.2330 & 153.02 & 0.2561 & 151.19 & 0.2781 & 151.46 & 0.1848 & 155.57 & 0.1895 & 167.47 \\
\hline 0.2021 & 147.67 & 0.2333 & 151.54 & 0.2468 & 155.56 & 0.2660 & 152.52 & 0.2195 & 157.31 & 0.1892 & 168.19 \\
\hline 0.1965 & 150.74 & 0.2361 & 150.53 & 0.2527 & 152.06 & 0.2588 & 154.30 & 0.1711 & 163.22 & 0.2351 & 138.76 \\
\hline 0.1898 & 152.21 & 0.2363 & 151.36 & 0.2573 & 150.58 & 0.2784 & 149.33 & 0.1977 & 151.91 & 0.1807 & 170.62 \\
\hline 0.1977 & 147.50 & 0.2318 & 153.04 & 0.2582 & 150.51 & 0.2719 & 152.87 & 0.1600 & 164.01 & 0.2449 & 138.57 \\
\hline 0.1799 & 154.58 & 0.2348 & 151.16 & 0.2522 & 153.64 & 0.2635 & 154.70 & 0.1969 & 156.12 & 0.1962 & 166.00 \\
\hline 0.1853 & 151.87 & 0.2291 & 153.07 & 0.2472 & 155.22 & 0.2675 & 153.61 & 0.1636 & 164.26 & 0.2379 & 138.62 \\
\hline 0.2038 & 146.84 & 0.2364 & 151.63 & 0.2518 & 153.17 & 0.2719 & 150.85 & 0.2043 & 155.88 & 0.1825 & 170.38 \\
\hline 0.1901 & 152.41 & 0.2382 & 150.25 & 0.2479 & 153.70 & 0.2625 & 153.12 & 0.2685 & 134.51 & 0.1893 & 167.50 \\
\hline \multirow[t]{12}{*}{0.1941} & 150.25 & 0.2338 & 151.66 & 0.2513 & 152.01 & 0.2679 & 151.38 & & & 0.2459 & 137.94 \\
\hline & & & & & & & & & & 0.1920 & 167.00 \\
\hline & & & & & & & & & & 0.2500 & 137.90 \\
\hline & & & & & & & & & & 0.2381 & 138.37 \\
\hline & & & & & & & & & & 0.1824 & 170.10 \\
\hline & & & & & & & & & & 0.1797 & 170.58 \\
\hline & & & & & & & & & & 0.2369 & 138.69 \\
\hline & & & & & & & & & & 0.1849 & 167.80 \\
\hline & & & & & & & & & & 0.2434 & 138.87 \\
\hline & & & & & & & & & & 0.1894 & 166.49 \\
\hline & & & & & & & & & & 0.2510 & 138.82 \\
\hline & & & & & & & & & & 0.2557 & 137.55 \\
\hline 0.1918 & 150.71 & 0.2340 & 151.81 & 0.2521 & 152.72 & 0.2677 & 152.92 & 0.1953 & 156.72 & 0.2465 & 138.25 \\
\hline
\end{tabular}


$\mathrm{CuZnMgAl}-\mathrm{NO}_{3}>\mathrm{CuZnMgAl}-\mathrm{F}>\mathrm{CuZnMgAl}-\mathrm{OH}>$ $\mathrm{CuZnMgAl}-\mathrm{Cl}>\mathrm{CuZnMgAl}-\mathrm{Br}>\mathrm{CuZnMgAl}-\mathrm{I}$, 与 CuZnMgAl-X 体系的总势能顺序一致; (2) 对比其 他体系, $\mathrm{CuZnMgAl}-\mathrm{NO}_{3}$ 的氢键强度最强, 在图 3 左上角和右下角均有分布, 原因是其层间阴离子 $\mathrm{NO}_{3}^{-}$中的 $\mathrm{N} 、 \mathrm{O}$ 均可作为氢键受体, 形成的氢键数 目最多; (3) CuZnMgAl-OH 的氢键数目虽然小于 $\mathrm{CuZnMgAl}-\mathrm{Cl} 、 \mathrm{CuZnMgAl}-\mathrm{Br} 、 \mathrm{CuZnMgAl}-\mathrm{I}$, 但是其 氢键强度却比它们大, 这主要是由于 $\mathrm{Cl}^{-} 、 \mathrm{Br}^{-} 、 \mathrm{I}^{-}$的离 子半径较大, 较难形成氢键所导致的; (4) 从计算所 得的氢键键长结果来看, 所形成的氢键键长总体比 一般的 $\mathrm{O}-\mathrm{H} \cdots \mathrm{O}$ 型氢键键长 $(0.240-0.276 \mathrm{~nm})$ 和 $\mathrm{O}-\mathrm{H} \cdots \mathrm{N}$ 型氢键键长 $(0.280-0.300 \mathrm{~nm})$ 均略短, 氢 键强度更强, 这主要是 LDHs 主客体间静电与氢键 协同效应的结果.

\section{5 态密度分析}

为了进一步了解 CuZnMgAl-X 体系中主客体 间的相互作用, 本文计算了 CuZnMgAl-X体系的单 独层板态密度、总态密度以及层间阴离子的分态密 度, 并将其用图表示出来(见图 4, 图 5).

图 4 为 $\mathrm{CuZnMgAl}-\mathrm{X}$ 体系单独层板的态密度分 布图. 从图中可以看出, CuZnMgAl-X体系主体层板 的态密度主要由四个区域组成: (1) 价带底部 $(-40--30 \mathrm{eV})$, 其态密度的贡献主要来自于 $\mathrm{Mg}$ 的 $2 p$ 轨道; (2) 价带中部 $(-25--17 \mathrm{eV})$, 其态密度贡

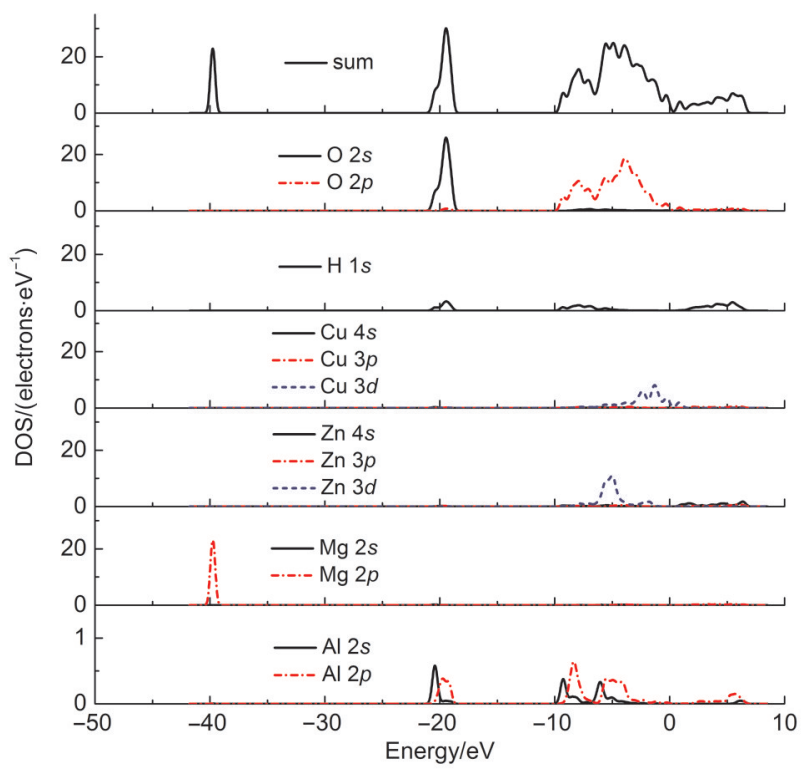

图 $4 \mathrm{CuZnMgAl-X}$ 体系主体层板的态密度(DOSs)

Fig.4 Densities of states (DOSs) of layer of the CuZnMgAl-X

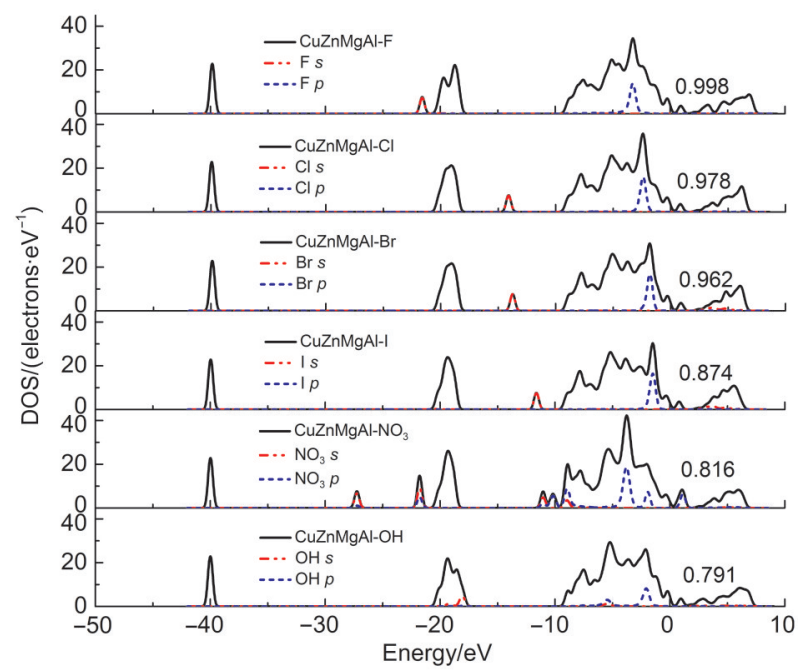

图 $5 \mathrm{CuZnMgAl}-\mathrm{X}$ 体系及其层间阴离子的态密度

Fig.5 DOSs of the CuZnMgAl-X and the anions

献主要来自于 $\mathrm{H}$ 的 $1 s$ 轨道, $\mathrm{O}$ 的 $2 s$ 轨道以及 $\mathrm{Al}$ 的 $3 s 、 3 p$ 轨道; (3) 价带顶部 $(-10-0 \mathrm{eV})$, 其态密度贡 献主要来自于 $\mathrm{O}$ 的 $2 p$ 轨道, $\mathrm{Zn}$ 的 $3 d$ 轨道, $\mathrm{Cu}$ 的 $3 d$ 轨道以及 $\mathrm{Al}$ 的 $3 s 、 3 p$ 轨道, $\mathrm{Zn}$ 的 $4 s 、 3 p$ 轨道和 $\mathrm{Cu}$ 的 $4 s 、 3 p$ 轨道也有部分贡献; (4) 导带底部 $(1-8 \mathrm{eV})$, 其 态密度贡献主要来自于 $\mathrm{H}$ 的 $1 s$ 轨道, $\mathrm{Cu}$ 的 $4 s 、 3 p$ 轨 道以及 $\mathrm{Zn}$ 的 $4 s 、 3 p$ 轨道.

图 5 为 $\mathrm{CuZnMgAl}-\mathrm{X}$ 体系的总态密度以及层间 阴离子的分态密度, 从图中可以看出以下几个特 征: (1) 当层间阴离子插层进入水滑石后, 层板 $\mathrm{O} 、 \mathrm{H}$ 之间成键作用逐渐减弱, 层板金属与层间阴离子之 间相互作用逐渐增强, 从而导致 CuZnMgAl-X 体系 比单独 $\mathrm{CuZnMgAl}$ 层板稳定性更强; (2) CuZnMgAl$\mathrm{X}$ 体系的禁带宽度较传统 MgAl-LDHs 的禁带宽度 $(2-5 \mathrm{eV})^{13,24,25}$ 小很多, 这主要是由于层板中 $\mathrm{Cu}$ 的掺 杂导致了LDHs 价带顶向导带底靠拢, 禁带宽度变 窄, 稳定性下降, 与文献 ${ }^{26-29}$ 报道的含铜水滑石较难 合成相符; (3) 对于 A 类和 B 类水滑石, 随着层间阴 离子电负性的减弱, 禁带宽度逐渐变窄, 体系电子 更易向高能级发生跃迁, 稳定性逐渐下降, 与结合 能分析结果相一致; (4) 对于 A 类和 B 类水滑石, 随 着层间阴离子电负性的减弱, 层间阴离子的 $s$ 轨道 和 $p$ 轨道总体向高能量处发生偏移, 这主要是由于 层间阴离子和层板间的氢键网络强度逐渐减弱所 导致的, 与氢键分析结果相一致.

\section{4 结 论}

采用噟势平面波法计算了 $\mathrm{CuZnMgAl}-\mathrm{X}(\mathrm{X}=$ 
$\mathrm{F}^{-}, \mathrm{Cl}^{-}, \mathrm{Br}^{-}, \mathrm{I}^{-}, \mathrm{OH}^{-}, \mathrm{NO}_{3}^{-}$) 的结构参数和电子性质, 探讨了不同层间阴离子对 CuZnMgAl-X 体系主客 体间超分子作用的影响, 结论如下:

(1) CuZnMgAl-X 体系主客体间存在着较强的 超分子作用力, 主要包括静电和氢键作用. 对于 $\mathrm{A}$ 类和 B类水滑石, 随着层间阴离子电负性的减弱, 电 子逐渐从层间阴离子向层板发生转移, 主客体间静 电作用力逐渐减小, 氢键强度逐渐降低, 两者协同 作用导致体系结合能逐渐下降, 稳定性降低.

(2) $\mathrm{CuZnMgAl}-\mathrm{X}$ 体系中同时存在着离子键与 共价键, 层板金属 $\mathrm{Mg} 、 \mathrm{Al}$ 的 $p$ 轨道和层间阴离子的 $s$ 轨道对体系的离子键部分贡献较大, 而层板金属离 子的 $s$ 轨道和层间阴离子的 $p$ 轨道对体系的共价键 部分贡献较大.

(3) $\mathrm{Cu}$ 等过渡金属的掺杂导致了 $\mathrm{CuZnMgAl}-\mathrm{X}$ 体系的价带顶向高能量处发生偏移, 禁带宽度较传 统 MgAl-LDHs 体系更窄, 稳定性更低. 对于 A 类和 B 类水滑石, 随着层间阴离子电负性的减弱, 禁带宽 度逐渐降低, 体系电子更易向高能级发生跃迁, 稳 定性逐渐下降。

\section{References}

(1) Cavani, F.; Trifiro, F.; Vaccari, A. Catal. Today 1991, 11, 173. doi: 10.1016/0920-5861(91)80068-K

(2) Duan, X.; Zhang, F. Z. Intercalation and Assembly Chemistry of Inorganic Supramolecular Materials; Science Press: Beijing, 2009. [段 雪, 张法智. 无机超分子材料的插层组装化学. 北京: 科学出版社, 2009.]

(3) Bellotto, M.; Rebours, B.; Clause, O.; Lynch, L. J. Phys. Chem. 1996, 100, 8535. doi: 10.1021/jp960040i

(4) Leuteritz, A.; Kutlu, B.; Meinl, J.; Wang, D.; Das, A.; Wagenknecht, U.; Heinrich, G. Mol. Cryst. Liq. Cryst. 2012, 556, 107. doi: 10.1080/15421406.2012.635923

(5) Asouhidou, D. D.; Triantafyllidis, K. S.; Lazaridis, N. K.; Matis, K. A. J. Chem. Technol. Biot. 2012, 87, 575. doi: 10.1002/jctb. v87.4

(6) Parida, K. M.; Mohapatra, L. Chem. Eng. J. 2012, 179, 131. doi: 10.1016/j.cej.2011.10.070

(7) Wang, S. L.; Huang, J. L.; Chen, F. S. China Pulp \& Paper 2012, 31, 14. [王松林, 黄建林, 陈夫山. 中国造纸, 2012, 31, 14.]

(8) Wu, J. S.; Xiao, Y. K.; Lin, Y. P.; Liang, H. Q.; Li, C. Y.; He, H. Y. Journal of Synthetic Crystals 2010, 39, 817. [吴健松, 肖应 凯, 林意萍, 梁海群, 李春银, 何海英. 人工晶体学报, 2010, 39, 817.]
(9) Wang, J. T.; Chen, L. P.; Zhan, Z. K. Chemical Research 2012, 23, 39. [王军涛, 陈兰萍, 詹正坤. 化学研究, 2012, 23, 39.]

(10) Heermann, D. W. Computer Simulation Methods in Theoretical Physics; Springer-Verlag Press: Heidelberg, 1990; pp 387-439.

(11) Leach, A. R. Molecular Modelling: Principles and Applications; Addison Wesley Longman Limitted Press: Essex, 2001; pp 26-454.

(12) Fraccarollo, A.; Cossi, M.; Marchese, L. Chem. Phys. Lett. 2010, 494, 274. doi: 10.1016/j.cplett.2010.06.029

(13) Xu, Q.; Ni, Z. M.; Mao, J. H. J. Mol. Struct. -Theochem 2009, 915, 122. doi: 10.1016/j.theochem.2009.08.033

(14) Wang, L. G.; Shi, W.; Yao, P.; Ni, Z. M.; Li, Y.; Liu, J. Acta Phys. -Chim. Sin. 2012, 28, 58. [王力耕, 施 炜, 姚 萍, 倪哲明, 李 远, 刘 娇. 物理化学学报, 2012, 28, 58.] doi: 10.3866/PKU.WHXB20122858

(15) Segall, M. D.; Linda, P.; Probert, M.; Pickard, C.; Hasnip, P.; Clark, S.; Payne, M. J. Phys. -Condes. Matter 2002, 14, 2717. doi: 10.1088/0953-8984/14/11/301

(16) Ceperley, D. M.; Aider, B. J. Phys. Rev. Lett. 1980, 45, 566. doi: 10.1103/PhysRevLett.45.566

(17) Vanderbilt, D. Phys. Rev. B 1990, 41, 7892. doi: 10.1103/ PhysRevB.41.7892

(18) Kresse, G.; Furthmiiller, J. Phys. Rev. B 1996, 54, 11169. doi: 10.1103/PhysRevB.54.11169

(19) Steven, G. B. J. Chem. Educ. 1985, 62, 101. doi: 10.1021/ ed062p101

(20) Mulliken, R. S. J. Chem. Phys. 1955, 23, 1833. doi: 10.1063/ 1.1740588

(21) Scheiner, S. Hydrogen Bonding; Oxford University Press: New York, 1997.

(22) Jeffrey, G. A. An Introduction to Hydrogen Bond; Oxford University Press: New York, 1997.

(23) Desiraju, G.; Steiner, T. The Weak Hydrogen Bond; Oxford University Press: New York, 1999.

(24) Hong, Y.; Min, W.; Jing, M.; Evans, D. G.; Xue, D. J. Phys. Chem. A 2010, 114, 7369. doi: 10.1021/jp9121003

(25) Cao, G. T.; Xu, Q.; Ni, Z. M. Acta Chim. Sin. 2011, 69, 2947. [曹根庭, 胥 倩, 倪哲明. 化学学报, 2011, 69, 2947.]

(26) Velu, S.; Suzuki, K.; Osaki, T. Catal. Lett. 1999, 62, 159. doi: 10.1023/A:1019023811688

(27) Morpurgo, S.; Jacono, M. L.; Porta, P. J. Solid State Chem. 1996, 122, 324. doi: 10.1006/jssc.1996.0121

(28) Ni, Z. M.; Yao, P.; Liu, X. M.; Wang, Q. Q.; Xu, Q. Chem. J. Chin. Univ. 2010, 31, 2438. [倪哲明, 姚 萍, 刘晓明, 王巧巧, 胥 倩. 高等学校化学学报, 2010,31,2438.]

(29) Liu, J.; Yao, P.; Ni, Z. M.; Li, Y.; Shi, W. Acta Phys. -Chim. Sin. 2011, 27, 2088. [刘 娇, 姚萍, 倪哲明, 李 远, 施 炜. 物理化学学报, 2011, 27, 2088.] doi: 10.3866/PKU. WHXB20110923 